\title{
Natürliche Mechanismen der Unkrautunterdrückung in ,Elstar'-Apfelanlagen
}

\author{
Antonia Engel ${ }^{1} \cdot$ Achim Kunz $^{1} \cdot$ Michael Blanke$^{1}$ \\ Eingegangen: 12. März 2020 / Angenommen: 14. November 2021 / Online publiziert: 14. Januar 2022 \\ (c) Der/die Autor(en) 2022
}

\section{Zusammenfassung}

Im Rahmen einer Masterarbeit sollte untersucht werden, warum der Baumstreifen in ausgewachsenen Elstar-Anlagen wesentlich weniger Aufwuchs aufweist als bei anderen Apfelsorten auf der gleichen Unterlage am gleichen Standort. Daher wurde die Vegetation in Baumstreifen von 'Elstar'-Anlagen im Vergleich zu drei anderen Apfelsorten in 150 Vegetationsaufnahmen an drei Terminen in einjährigen Untersuchungen am Campus Klein-Altendorf bonitiert. Daneben wurde die Lichtdurchlässigkeit der Baumkrone mittels 300 Ceptometer- und 100 Schachbrettmessungen der Sonnenflecken im Baumstreifen im Juni und September - nach der Herbizidapplikation im Mai 2019 - ermittelt.

Die mit der Schachbrettmethode bzw. mit dem Ceptometer ermittelte Lichteinstrahlung durch die Baumkrone auf den Baumstreifen unter den 'Elstar'-Apfelbäumen war im September um ca. $35 \%$ bzw. ca. $10 \%$ signifikant geringer als unter Bäumen gleichen Alters der Sorte 'Golden Delicious' in der Nachbarreihe auf der gleichen Unterlage M 9.

Der Baumstreifen unter ausgewachsenen 'Gala'- und 'Braeburn'-Apfelbäumen wies einen statistisch signifikant höheren Bodenbedeckungsgrad mit Beikräutern mit Boniturnoten von 3,8-4,3 im Vergleich zu 1,7-1,9 bei 'Elstar' auf (auf der Skala 1-5) - auf zwei nach IP-Richtlinien und einer biologisch bewirtschafteten Fläche, d.h. die Art der Baumstreifenbehandlung (chemisch oder mechanisch) hatte keinen Einfluss auf das Phänomen der Beikrautunterdrückung bei ausgewachsenen 'Elstar'-Apfelbäumen.

Sowohl Kreuzkraut (Senecio spp.) als auch Gänseblümchen (Bellis perennis) traten in den Baumstreifen unter ausgewachsenen 'Elstar'-Apfelbäumen mit wesentlich geringerer Stetigkeit auf als unter 'Golden Delicious' in der benachbarten Reihe. Die in der Literatur beschriebene indirekte Selektion durch langjährige Glyphosatanwendung auf Vogelmiere (Stellaria media) und Kreuzkraut (Seneccio spp.) durch Auskeimen der Samen nach der Herbizidapplikation wurde bestätigt; im vorliegenden Versuch traten jedoch Behaartes Schaumkraut (Cardamine hirsuta) und Rispengräser (Poa spp.) stärker auf. Keimtests in Aussaatschalen - aufgestellt in den Baumstreifen - unter den Apfelbäumen der beiden Apfelsorten 'Elstar, Michielsen' und als Vergleich 'Golden Delicious' mit einem Licht- (Lepidium sativum) und einem Dunkelkeimer (Borago officinalis) zeigten keinen Unterschied in der Keimrate. Keimtests mit Kressesamen (Lepidium sativum) in mit Falllaub vermischtem Substrat in Aussaatschalen im Gewächshaus zeigten keine keimhemmende und damit keine allelopathische Wirkung des Falllaubes der Sorte 'Elstar'.

Die vorliegenden Ergebnisse der Beikrautunterdrückung im Wiederaufwuchs nach Beikrautbekämpfung stimmen mit denen unter starkwüchsigen 'Ingrid Marie' auf M 2 aus der Literatur überein. Diese Wirkung ausgewachsener Apfelbäume der Sorte 'Elstar, Michielsen' auf M 9 ist durch die Schattierung dieser wüchsigeren Bäume bedingt und hängt nicht von der Bewirtschaftungsform (IP mit Herbizideinsatz oder Öko mit mechanischer Beikrautbekämpfung), aber eventuell noch von weiteren Faktoren, ab.

Schlüsselwörter Apfel (Malus domestica Borkh) · Baumstreifen · Beschattung · Glyphosatersatz · Herbizid · Herbizidselektion $\cdot$ Lichteinstrahlung $\cdot$ Nachhaltigkeit $\cdot$ Unkraut $\cdot$ Vegetationskartierung

Michael Blanke

mmblanke@uni-bonn.de

1 INRES-Gartenbauwissenschaft, Universität Bonn, Bonn,

Deutschland 


\title{
Natural Mechanisms of Weed Suppression of cv. 'Elstar' Apple Trees
}

\begin{abstract}
The objective of this field trial was to investigate causes of the reduced weed population/vegetation in the herbicide strip of fully-grown cv. 'Elstar' apple trees. The vegetation in the herbicide strip of cv. 'Elstar' with 150 assessments was compared with that under cv. 'Golden Delicious', 'Gala' and 'Braeburn' apple trees on the same rootstock (M 9) at the same location at Klein-Altendorf, University of Bonn, Germany $\left(51^{\circ} \mathrm{N}\right)$. To determine the amount of light available to the vegetation in the herbicide strip, light interception of the tree canopy was measured. The 300 ceptometer and the 100 sunfleck measurements with the 'point quadrat method' (chessboard) in September after herbicide application in May showed a significant $10 \%$ (ceptometer) and 35\% (sunfleck) light reduction in the herbicide strip under 'Elstar' apple trees relative to 'Golden Delicious' in the adjacent tree row.

The tree strip under fully-grown cv. 'Gala' and 'Braeburn' apple trees was statistical significantly more densely covered with weeds rated as 3.8-4.3 in comparison with that of cv. 'Elstar' rated as 1.7-1.9 on a scale of $1(0-20 \%)$ to $5(80-100 \%$ surface covered); this finding applied to both chemical (IP) and mechanical (organic) weeding.

Common groundsel (Senecio spp.) and daisy (Bellis perennis) were less abundant in the tree strips under fully-grown 'Elstar' apple trees than under fully-grown 'Golden Delicious' apple trees of the adjacent row. The indirect weed species selection as a result of longtime glyphosate application as described in the literature was confirmed; this herbicide application allowed the subsequent germination of seeds of common chickweed (Stellaria media) and common groundsel (Seneccio spp.). In the present experiment, cuckooflower (Cardamine hirsuta) and meadow grasses (Poa spp.) dominated.

Seeds in trays placed in the strip under the more shading cv. 'Elstar' and the less shading cv. 'Golden Delicious' tree canopy showed no difference in germination rate. Similarly, seeds of garden cress (Lepidium sativum) showed no difference in germination rate in trays with substrate mixed with cv. 'Elstar' or cv. 'Golden Delicious' leaves (1/10 v/v) thereby discounting an allelopathic cause.

The present results of weed suppression by the cv. 'Elstar' trees after herbicide application are in line with those reported of vigorous apple cv. 'Ingrid Marie' on M2 rootstock in the literature in the same region. This weed suppression by fully-grown cv. 'Elstar, Michielsen' appears not only cultivar dependent, but may be related to shading by the canopy of vigorous fruit trees, irrespective of cultivation (IFP with herbicide or organic production with mechanical weeding) and possibly influenced by further factors.
\end{abstract}

Keywords Apple (Malus domestica Borkh) · Ceptometer · Glyphosate substitute · Seed Germination · Herbicide $\cdot$ Light . MCPA $\cdot$ Sustainability $\cdot$ Vegetation $\cdot$ Weed

\section{Einleitung}

Frühere pflanzensoziologische Studien konzentrierten sich auf die Erforschung der selektiven Wirkung verschiedener Herbizide auf die Zusammensetzung der Unkraut- bzw. Begleitflora in Wein- und Obstanlagen (Engel 1968; Kolbe 1969; Nohl 1982; Stalder et al. 1977). Bei diesen Vegetationskartierungen stellte sich heraus, dass wiederholte Glyphosatanwendung sowohl im Weinbau (Stalder et al. 1977) als auch im Apfelanbau im Meckenheimer Obstbaugebiet einjährige Samenunkräuter wie Vogelmiere (Stellaria media) und Kreuzkraut (Senecio vulgaris) selektierte, wohingegen Gemeine Quecke (Agropyron repens), Weißes Straußgras (Agrostis stolonifera), Kriechender Hahnenfuß (Ranunculus repens) und Ampfer (Rumex spp.) verdrängt wurden, während der Zusatz von MCPA Einjährige Rispengräser (Роа аппиа) selektierte (Nohl 1982).

In der Obstbaupraxis wurde beobachtet (K. J. Wiesel, persönliche Mitteilung), dass Baumstreifen unter ausgewachsenen 'Elstar'-Bäumen, der mit ca. 6700 ha Anbau- fläche in Deutschland am häufigsten angebauten Apfelsorte (Statistisches Bundesamt 2017), auf M 9 eine geringere Verunkrautung aufweisen als Baumstreifen unter Bäumen gleichen Alters anderer Apfelsorten auf der gleichen Unterlage. Da bei diesen Beobachtungen Glyphosat und MCPA zum Einsatz kam, beruht dieses Phänomen nicht unbedingt nur auf einer selektiven Wirkung des verwendeten Herbizids.

Bereits 1969 beobachtete Kolbe im Rheinland einen geringeren Wiederaufwuchs der Beikräuter nach der Herbizidapplikation im Frühjahr durch die stärker beschattende Apfelsorte 'Ingrid Marie' auf der Unterlage M2 im Vergleich zu kleineren Bäumen von 'James Grieve' auf der gleichen Unterlage; beobachtet wurde eine geringere Wiederbesiedelung des Baumstreifens mit Beikräutern im Herbst nach Applikation der Herbizide im Frühling.

Ziel der vorliegenden Arbeit war es daher, mögliche Ursachen dieses Phänomens des geringeren Aufwuchses in beschatteten Baumstreifen zu untersuchen. Dazu wurden ca. $1,5 \mathrm{~m}$ breite Baumstreifen in drei Parzellen mit 'El- 
star' und anderen Apfelsorten zum Vergleich im Rahmen einer Masterarbeit im Verlauf der Vegetationsperiode 2019, vor und nach der Herbizidapplikation, untersucht. Vegetationskartierungen erfolgten sowohl im Baumstreifen mit mechanischer Beikrautbekämpfung als auch mit Herbizideinsatz. Zusätzlich wurden Messungen der Beschattung im Baumstreifen mittels Schachbrettmethode und Ceptometer sowie zwei Keimtests durchgeführt.

Für die Untersuchungen wurde kein neuer Versuch angelegt, weil das Phänomen der Beikrautunterdrückung 1. bei ausgewachsenen 'Elstar'-Apfelbäumen auf gleicher schwachwüchsiger Unterlage M 9 wie bei den Vergleichsbäumen auftritt und 2. jeder Boden bzw. Standort sein eigenes Samenreservoir mitbringt, dessen Vorkommen unbekannt ist und hier absichtlich nicht beeinflusst wurde. Dies muss bei der Interpretation der vorliegenden Ergebnisse berücksichtigt werden.

\section{Material und Methoden}

\section{Standort, Klima und Boden}

Die Untersuchungen erfolgten auf dem Campus Klein-Altendorf der Universität Bonn. Der Standort ist geprägt durch ein gemäßigtes Klima mit atlantischen Winden und einer Jahresdurchschnittstemperatur von $9,8^{\circ} \mathrm{C}$ und $603 \mathrm{~mm}$ Niederschlag pro Jahr. Angaben zu Bodenparametern finden sich in Tab. 1.

\section{Apfelbäume}

In die Untersuchung wurden alle Parzellen mit ausgewachsenen 'Elstar'-Apfelbäumen des Versuchsguts einbezogen (Tab. 2). Bei der ersten Parzelle (C2) waren die Apfelbäume der drei Sorten 'Elstar', 'Gala' und 'Braeburn' in Blöcken quer zu den Reihen angeordnet, sodass in einer Reihe drei Sorten hintereinander wuchsen (Abb. 1).

Im Gegensatz dazu waren bei den anderen beiden Versuchsparzellen (J1 und F2) jeweils vier Reihen 'Elstar' durch eine Reihe 'Golden Delicious' als Befruchter getrennt. Alle IP-Parzellen waren im Vorjahr einmal mit Glyphosat und MCPA (Juni 2018) behandelt worden (Tab. 2).

Tab. 1 Bodenparameter am Standort Klein-Altendorf in $0-25 \mathrm{~cm}$ Tiefe (Ap-Horizont)

\begin{tabular}{ll}
\hline Parameter & Wert \\
\hline Bodenart & Lehmiger Schluff \\
Bodentyp & Parabraunerde \\
pH-Wert & $\mathrm{pH} \mathrm{6,8}$ \\
Org. Substanz & $1,8 \%$ \\
Bodenpunkte & 93 \\
\hline
\end{tabular}
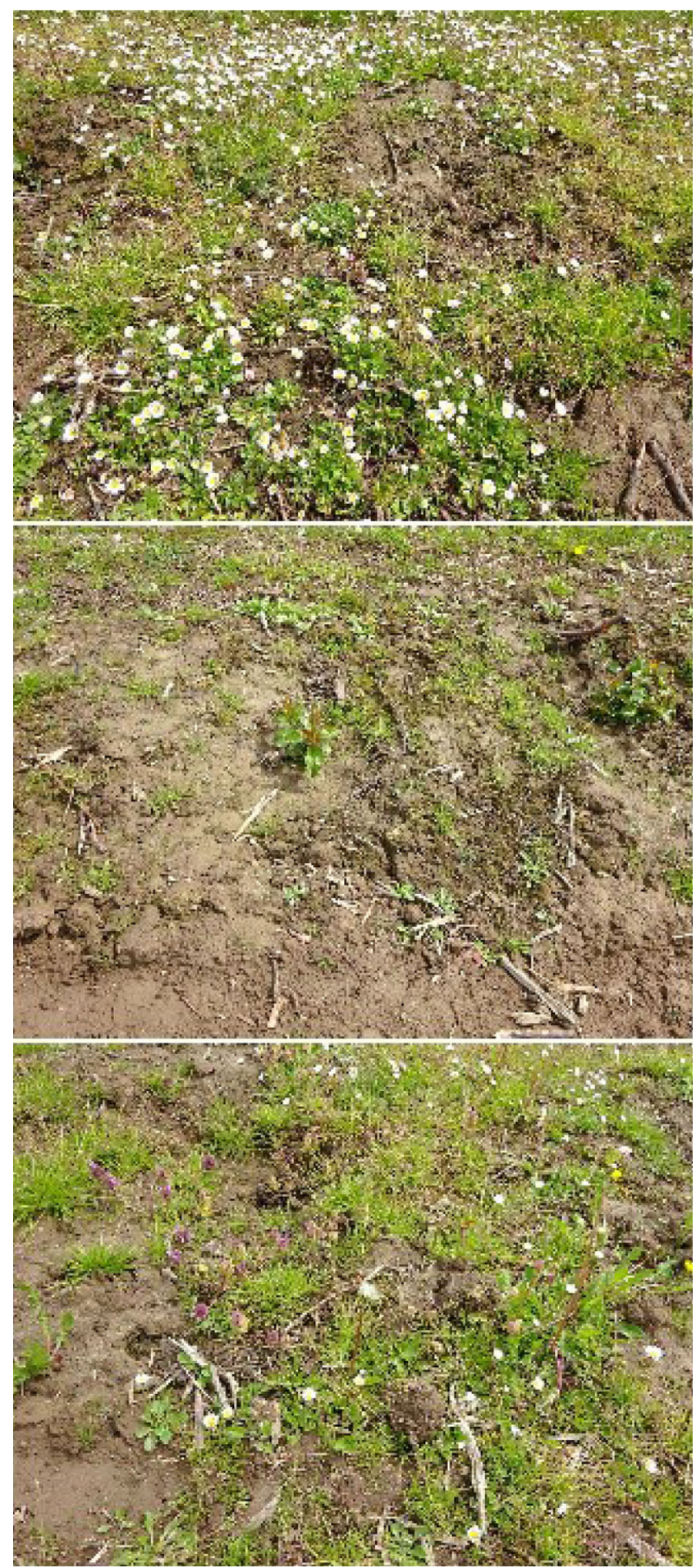

Abb. 1 Vegetation im Baumstreifen von gerodeten 'Gala, Mondial' (oben), 'Elstar, Michielsen' (Mitte) und 'Braeburn, Mariri Red'-Apfelbäumen (unten) innerhalb derselben Reihe auf der Fläche C2 am Campus Klein-Altendorf im Mai 2019 
Tab. 2 Übersicht über die untersuchten Parzellen am Campus Klein-Altendorf

\begin{tabular}{llll}
\hline Parameter & C2 & J1 & F2 \\
\hline Unterlage & $\begin{array}{l}\text { M 9 } \\
\text { (gerodet im Februar 2019) }\end{array}$ & M 9 & M \\
Vergleichssorte & $\begin{array}{l}\text { Gala (Mondial), Braeburn (Mariri } \\
\text { Red) }\end{array}$ & Golden Delicious & Golden Delicious \\
Pflanzjahr & 2007 & 2011 & 2013 \\
Hagelnetze & Hagelnetz & Hagelnetz & Kein Hagelnetz \\
Baumstreifenbehandlung 2017 & Mechanisch (Öko) & - & - \\
& $\begin{array}{l}\text { a. Herbizid Ende April (IP) Gly- } \\
\text { phosat und MCPA }\end{array}$ & a. Herbizid Ende April: & a. Herbizid Ende April: \\
& b. Herbizid im Herbst (IP) Basta & Glyphosat und MCPA & Glyphosat und MCPA \\
& bnd MCPA & und MCPA & b. Herbizid im Herbst: Basta \\
und MCPA & - \\
Baumstreifenbehandlung im & Mechanisch (Öko) & - & Herbizid Juni: \\
Vorjahr (2018) & Herbizid Juni (IP): & Herbizid Juni: & Glyphosat und MCPA \\
Baumstreifenbehandlung im & Keine & Glyphosat und MCPA & Herbizid Mai 2019: \\
laufenden Jahr & In einer Reihe & Herbizid Mai 2019: & Glyphosat und MCPA \\
Anordnung der Sorten & & Nachbarreihe & Nachbarreihe \\
\hline
\end{tabular}

\section{Bonituren und Messungen}

\section{Sonnenflecken als Maß des Beschattungsgrades}

Um die Beschattungsintensität in den Baumstreifen unter den Apfelbäumen zu erfassen, wurden in Anlehnung an Warren Wilson (1960) die Sonnenflecken im Baumstreifen $4 \times$ an einem Tag ohne Wolken an den in Tab. 3 aufgeführten Terminen bestimmt. Dabei kam ein $50 \mathrm{~cm} \times 50 \mathrm{~cm}$ großes selbst konstruiertes Schachbrett, im englischen als point quadrat method bezeichnetes Verfahren zum Einsatz. Die Feldgröße betrug $5 \mathrm{~cm} \times 5 \mathrm{~cm}$, sodass das Schachbrett aus 100 Feldern bestand (Abb. 2) und die Werte damit automatisch in Prozent sind. Die Zählung der Sonnenflecken wurden in den Parzellen J1 und F2 an denselben (18- bis 25mal pro Reihe) Stellen durchgeführt, wie die Bonitur der Vegetation. Um den Beschattungsgrad zu ermitteln, wurden die Sonnenflecken auf der sonnenabgewandten Seite bestimmt, d.h. vormittags auf der West- und nachmittags auf der Ostseite der Baumreihe. Das Schachbrett wurde immer rechts vom Baum platziert, so dass ein Viertel der Fläche abgedeckt war, die bei den Vegetationskartierungen erfasst wurde (Abb. 2a). Es wurde der prozentuale Anteil an Feldern mit Sonnenflecken (die mindestens 30\% des Feldes ausfüllten) ausgewertet, d.h. der Felder mit direkter Sonneneinstrahlung auf dem Boden unterhalb des Baumes.

\section{Ceptometer-Messungen}

Die Messungen des Beschattungsgrades mit der Schachbrettmethode wurden begleitet von Lichtmessungen mit dem Ceptometer vom Typ SunScan SS1 (Fa. Delta T Devices, Cambridge, England). Das Ceptometer besteht aus 56 PAR-Lichtsensoren, und einem Referenzsensor auf einem Dreifuß (Abb. 2d). Das Ceptometer wurde parallel zur Baumreihe auf dem Schachbrett platziert (Abb. 2c). Pro Baum wurden 10 Messungen, d.h. eine Messung pro Schachbrettreihe und zehn Messungen pro Sorte (d.h. 100

Tab. 3 Zeitlicher Versuchsablauf in Klein-Altendorf 2019

\begin{tabular}{lll}
\hline Vegetationskartierung & Lichtmessungen & Termin \\
\hline 1. Bonitur der Vegetation & - & 9. Mai 2019 \\
Herbizidbehandlung & Lichtmessung (Schachbrett) & 18. Mai 2019 \\
& - & 24. Juni 2019 \\
2. Bonitur der Vegetation & - & 11. Juli 2019 \\
Anlegen des 1. Keimversuchs & - & 20. August 2019 \\
Bonitur des 1. Keimversuchs & 1. Lichtmessung mit Ceptometer und Schachbrettmessung & 30. August 2019 \\
& 2. Lichtmessung mit dem Ceptometer & 30. August 2019 \\
3. Bonitur der Vegetation & - & 26. September 2019 \\
Anlegen des 2. Keimversuchs & - & 14. Oktober 2019 \\
Bonitur des 2. Keimversuchs & - & 13. November 2019 \\
\hline
\end{tabular}



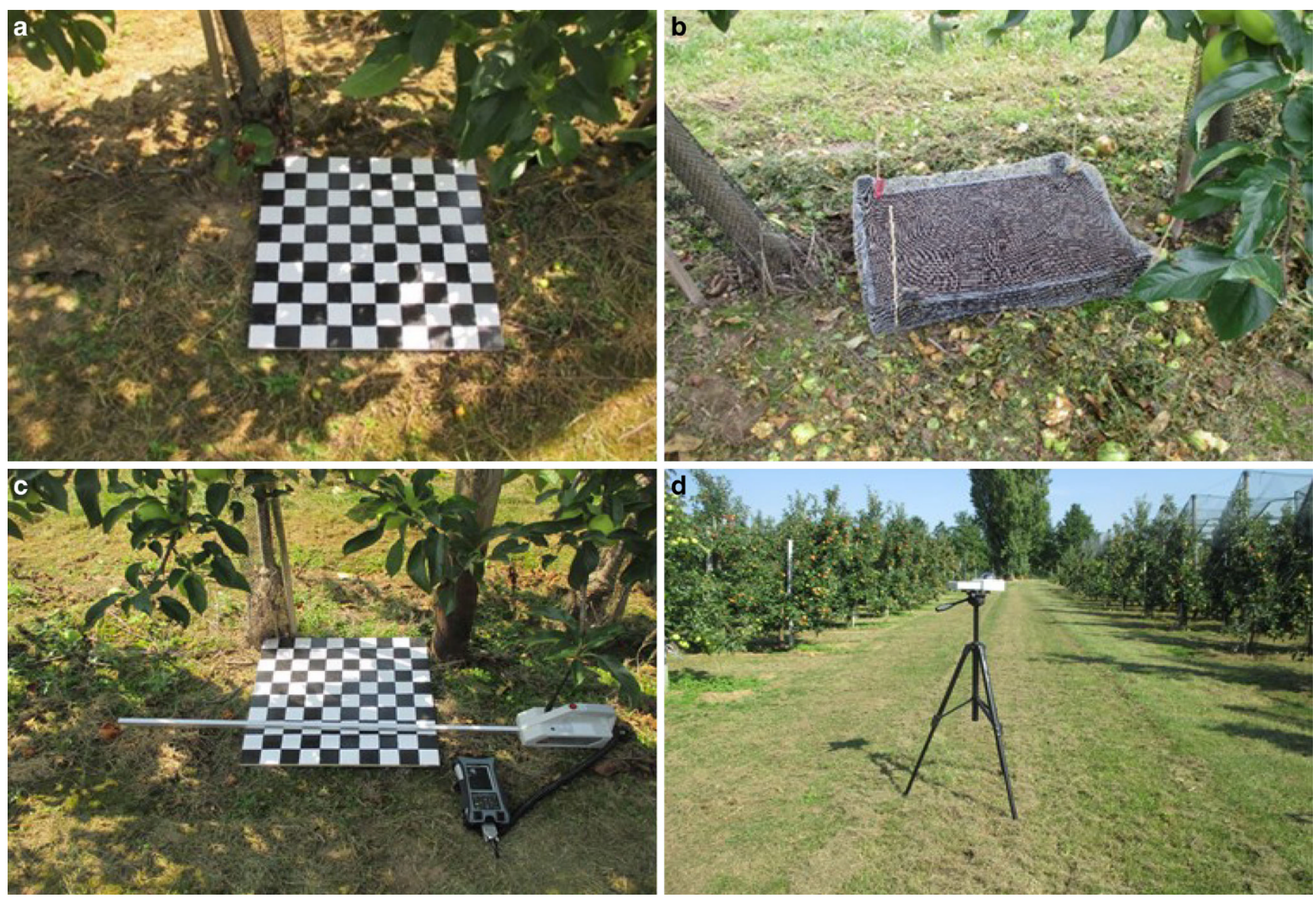

Abb. 2 a Bestimmung der Lichtverfügbarkeit mit der point quadrat method unter Apfelbäumen am Campus Klein-Altendorf (links oben); b Keimtest mit dem Lichtkeimer Kresse (Lepidium sativum) und dem Dunkelkeimer Borretsch (Borago officinalis) unter den 'Elstar'-Apfelbäumen (rechts oben); c Position des Ceptometers auf dem Schachbrett zur Messung der Lichteinstrahlung im Baumstreifen (links unten); d Referenzsensor für die Ceptometer-Messungen am Ende der Baumreihe (rechts unten)

Messungen pro Sorte und Parzelle und Messdurchgang) an den gleichen Terminen, wie die zweite Schachbrettmessung, durchgeführt. Der Referenzsensor auf dem Dreifuß wurde so weit außerhalb des Hagelnetzes am Ende der Reihe platziert, dass eine Beschattung vermieden wurde (Abb. 2d).

\section{Vegetationskartierung}

Die Vegetationskartierung in den Baumstreifen erfolgte nach internationalem Standard (Nohl 1982) in einem $1 \mathrm{~m}^{2}-$ Messrahmen. Pro Termin und Reihe wurden 12-16 (J1: 12,

Tab. 4 Eigenes Boniturschema zur Erfassung des Bodenbedeckungsgrads und der Wuchsstärke mit Beikräutern

\begin{tabular}{llll}
\hline Bedeckungsgrad [\%] & Boniturnote & Wuchsstärke & Boniturnote \\
\hline $0-20$ & 1 & Gering & 1 \\
$20-40$ & 2 & Mittel & 2 \\
$40-60$ & 3 & Stark & 3 \\
$60-80$ & 4 & - & - \\
$80-100$ & 5 & - & - \\
\hline
\end{tabular}

F2: 13; C2: 16) Messrahmen mithilfe eines Boniturschemas (Tab. 4) in Anlehnung an Braun-Blanquet (1964) ausgewertet, sodass insgesamt 150 Vegetationskartierungen an drei Terminen erfolgten. Bonitiert wurden am 9. Mai, 11. Juli und 14. Oktober 2019 (Tab. 3) der Bedeckungsgrad und die ca. 10 dominierenden Pflanzenarten in den Baumstreifen. Die erste Bonitur erfolgte im Mai vor und die zweite und die dritte nach der Herbizidapplikation im Mai 2019.

\section{Keimtest}

Um den Einfluss der Lichtdurchlässigkeit der Baumkrone auf die Wiederbesiedelung der Baumstreifen zu untersuchen (Werth et al. 2019), wurden zwei Keimtests durchgeführt (Abb. 2b). Dazu wurden je 200 Samen des Lichtkeimers Kresse (Lepidium sativum) und des Dunkelkeimers Borretsch (Borago officinalis) in Saatschalen mit einem kommerziellen Anzuchtsubstrat (Einheitserdewerke Werkverband e. V.) im August nach der Methode von Lepsis und Blanke $(2001,2005)$ unter den Bäumen zur Keimung gebracht und die prozentuale Keimrate bestimmt. 
Im zweiten Keimversuch wurden am 13. November frisch geerntete Apfelblätter der Sorte 'Elstar, Michielsen' und als Vergleich Blätter von 'Golden Delicious' mit dem Substrat vermischt (Verhältnis 1:10 (v/v)) und die Wirkung auf die Keimung von Samen der Gartenkresse (Lepidium sativum) im Gewächshaus in Anlehnung an Lepsis und Blanke $(2001,2005)$ am 17. Dezember bonitiert (Kressetest).

\section{Statistische Auswertung}

Die statistische Auswertung der Messergebnisse erfolgte in Excel mit zweifaktorieller (Sorte mal Bewirtschaftungsform) Varianzanalyse ANOVA. Die Messergebnisse wurde auf Normalverteilung und Varianzhomogenität geprüft. Angegeben sind die Mittelwerte und Standardfehler (SE) bei $5 \%$ Fehler-Wahrscheinlichkeit.

\section{Ergebnisse}

Ziel der vorliegenden Arbeit war es, mögliche natürliche Wirkungsmechanismen der Beikrautunterdrückung bei 'Elstar'-Apfelbäumen zu untersuchen; dazu wurden im ersten Schritt die dominierenden Pflanzenarten auf ihr Vorkommen und ihre Verbreitung bonitiert (Tab. 5).

\section{Vegetationskartierung in der Parzelle C2}

Abb. 3 zeigt den signifikant höheren Bodenbedeckungsgrad der Baumstreifen mit Beikräutern und Boniturnoten von 3,8 bis 4,3 bei 'Gala' und 'Braeburn' im Vergleich zu 1,7 bis 1,9 bei 'Elstar'. Diese unterschiedlichen Bedeckungsgrade wurden auf nach IP-Richtlinien und ökologisch bewirtschafteten Flächen beobachtet, d.h. die Anbauform bzw. Baumstreifenbehandlung (chemisch oder mechanisch) hat-

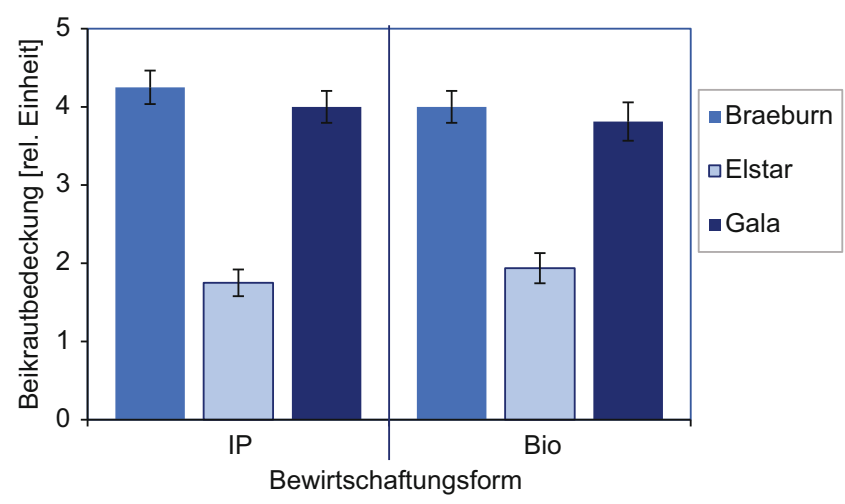

Abb. 3 Beikrautbedeckungsgrad unter gerade gerodeten Apfelbäumen der Sorten 'Elstar, Michielsen', 'Gala, Mondial' und 'Braeburn, Mariri Red' auf dem Campus Klein-Altendorf auf nach IP-Richtlinien (chemische Unkrautbekämpfung) und biologisch (mechanische Unkrautbekämpfung) bewirtschafteten Flächen zum ersten Boniturtermin am 9. Mai 2019. $\mathrm{MW} \pm \mathrm{SE}(n=16)$

te keinen Einfluss auf das Phänomen der Beikrautunterdrückung bei Elstar Apfelbäumen.

\section{Vegetationskartierungen in der Parzelle C2}

Abb. 4 zeigt die unterschiedliche Zusammensetzung der Beikrautflora in Baumstreifen mit mechanischer Unkrautbekämpfung und Herbizidanwendung unter Bäumen der Apfelsorten 'Elstar, Michielsen', 'Gala, Mondial' und 'Braeburn, Mariri Red'.

\section{Vegetationskartierung in der Parzelle F2}

Die Vegetationskartierung am 9. Mai 2019 vor der Herbizidapplikation am 18. Mai 2019 zeigt sowohl die indirekte Selektion der Beikräuter durch Glyphosat auf Vogelmiere (Stellaria media), Kreuzkraut (Seneccio spp.) und Taubnessel (Lamium spp.) (Tab. 5 und 6), als auch die dominierenden Arten, allen voran Löwenzahn (Taraxcacum of-

Tab. 5 Selektierte Pflanzenarten im Baumstreifen der Apfelanlagen in Klein-Altendorf eingeteilt nach der selektiven Langzeitwirkung durch Glyphosat

\begin{tabular}{|c|c|c|c|c|c|c|}
\hline Deutscher Name & Wissenschaftlicher Name & Blüte & Samenunkraut & Wurzelunkraut & Lichtkeimer & Dunkelkeimer \\
\hline \multicolumn{7}{|c|}{ Selektierte Arten (Literaturangaben) } \\
\hline Vogelmiere & Stellaria media & $\mathrm{Ja}$ & $\mathrm{Ja}$ & Nein & $\mathrm{Ja}$ & Nein \\
\hline Taubnessel & Lamium spp & $\mathrm{Ja}$ & $\mathrm{Ja}$ & Nein & k. A. & k. A. \\
\hline Gewöhnliches Kreuzkraut & Senecio vulgaris & $\mathrm{Ja}$ & $\mathrm{Ja}$ & Nein & k. A. & k. A. \\
\hline \multicolumn{7}{|l|}{ Andere vorkommende Arten } \\
\hline Behaartes Schaumkraut & Cardamine hirsuta & $\mathrm{Ja}$ & $\mathrm{Ja}$ & Nein & k. A. & k. A. \\
\hline Gänseblümchen & Bellis perennis & $\mathrm{Ja}$ & $\mathrm{Ja}$ & Nein & $\mathrm{Ja}$ & Nein \\
\hline Löwenzahn & Taraxacum officinale & $\mathrm{Ja}$ & $\mathrm{Ja}$ & $\mathrm{Ja}$ & $\mathrm{Ja}$ & Nein \\
\hline Ehrenpreis & Veronica spp & $\mathrm{Ja}$ & $\mathrm{Ja}$ & Nein & $\mathrm{Ja}$ & $\mathrm{Ja}$ \\
\hline Gänsedistel & Sonchus spp & Nein & $\mathrm{Ja}$ & $\mathrm{Ja}$ & $\mathrm{Ja}$ & Nein \\
\hline Gräser & Poa spp & Nein & $\mathrm{Ja}$ & Nein & $\mathrm{Ja}$ & Nein \\
\hline
\end{tabular}

k. A. keine Angabe 
Abb. 4 Häufigkeit (Anzahl der Boniturrahmen in denen die Beikrautarten nachgewiesen wurden) der Beikrautarten auf dem ökologisch und nach IPRichtlinien bewirtschafteten Teil des Schlags C2 am Campus Klein-Altendorf der Universität Bonn im Baumstreifen unter Apfelbäumen der Sorten 'Braeburn Mariri Red', 'Gala Mondial' und 'Elstar Michielsen' bonitiert am 9. Mai 2019 vor der Herbizidapplikation am 18. Mai 2019

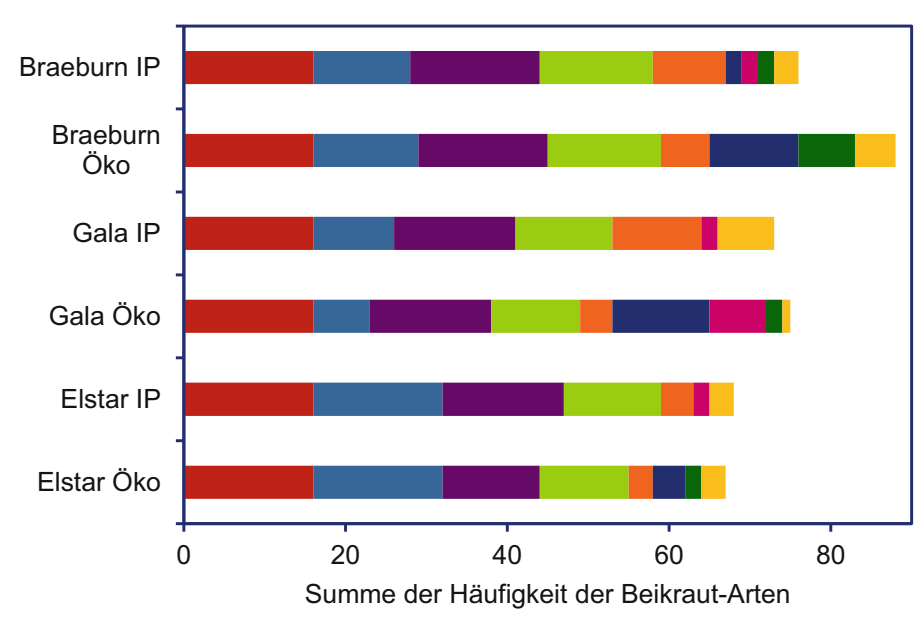

ficinale), Behaartes Schaumkraut (Cardamine hirsuta) und Gräser (Poa spp.) (Abb. 5a).

Abb. 5b zeigt die Boniturergebnisse der gleichen Parzelle zwei Monate nach der Herbizidapplikation, d.h. die geringere Verbreitung der Beikräuter im Baumstreifen unter den ausgewachsenen 'Elstar'-Apfelbäumen im Vergleich zum Baumstreifen der Nachbarreihe mit 'Golden Delicious' als Bestäuber; beide Sorten stehen auf der Unterlage M 9 und die Bäume haben das gleiche Alter (Abb. 5b). Sowohl Kreuzkraut (Senecio spp.) als auch Gänseblümchen (Bellis perennis) traten in den Baumstreifen unter 'Elstar' mit wesentlich geringerer Stetigkeit auf als unter 'Golden Delicious' in der benachbarten Reihe. Vogelmiere (Stellaria media), Ehrenpreis (Veronica spp.) und Taubnessel (Lamium spp.), deren Samen nach einem Monat noch nicht aufgelaufen sind, waren in dieser heißen Sommerperiode (bis $41^{\circ} \mathrm{C}$ ) besonders betroffen von der Kombination aus Beikrautunterdrückung durch 'Elstar' und Glyphosatanwendung einen Monat zuvor (Abb. 5b). Bei der Herbstbonitur zeigte sich jedoch wieder ein stärkerer Aufwuchs von Kreuzkraut ( $\mathrm{Se}$ neccio spp.) und Taubnessel (Lamium spp.) (Abb. 5c).

Abb. 6 zeigt den mittleren Beikrautbedeckungsgrad und die Wuchsstärke, angegeben jeweils als Boniturnote nach Tab. 4. Sowohl der Bedeckungsgrad als auch die Wuchsstärke der Beikräuter im Baumstreifen bei 'Golden Delicious' wurde bei der Vegetationskartierung im Mai 2019 vor der Herbizidapplikation mit den Boniturnoten 3,1 bzw.
2,6 bewertet und überstieg damit signifikant den Wert bei 'Elstar' mit 2,2 bzw. 2,2 (Abb. 6) und zeigt den geringeren Unkrautbesatz unter 'Elstar' Apfelbäumen (in dieser Parzelle).

Nach der Herbizidapplikation im Mai 2019 überstieg die mittlere Anzahl der unterschiedlichen Beikrautarten pro Boniturrahmen im Baumstreifen von 'Golden Delicious' erst die der Anzahl bei 'Elstar'; dieser Unterschied glich sich mit zunehmendem Aufwuchs im Herbst auf das Niveau der Frühlingswerte an (Abb. 7).

\section{Messung des Beschattungsgrades in der Parzelle J1}

Nach Ergebnissen von Wagenmakers und Tazelaar (1999) in Wilhelminadorp (Zeeland) wurden die Lichtmessungen in der von Palmer und Jackson (1977) empfohlenen Nord-Süd ausgerichteten Baumreihe im Juni 2019 um 10:30 Uhr und 14:45 Uhr und im Juli um 10:40 Uhr und 14:30 Uhr durchgeführt, weil zu diesem Zeitpunkt Sonnenflecken und simulierter Lichteinfall nach Wagenmakers und Tazelaar (1999) an unserem $51^{\circ}$ Breitengrad am besten korrelierten.

Abb. 8 zeigt eine $25 \%$ bis $30 \%$ signifikant geringere Lichteinstrahlung im Juni 2019 unter den 'Elstar'-Baumkronen als unter Apfelbäumen gleichen Alters der Sorte 'Golden Delicious' in der Nachbarreihe, besonders deutlich zeigte sich der Unterschied am frühen Morgen, wenn

Tab. 6 Unterrepräsentierte Pflanzenarten in den Apfelanlagen in Klein-Altendorf

\begin{tabular}{|c|c|c|c|c|c|c|}
\hline Deutscher Name & Wissenschaftlicher Name & Blüte & Samenunkraut & Wurzelunkraut & Lichtkeimer & Dunkelkeimer \\
\hline$\overline{\text { Melde }}$ & Atriplex spp & Nein & $\mathrm{Ja}$ & Nein & k. A. & k. A. \\
\hline Schafgarbe & Achillea millefolium & Nein & Nein & $\mathrm{Ja}$ & $\mathrm{Ja}$ & Nein \\
\hline Sauerampfer & Rumex acetosella & $\mathrm{Ja}$ & Nein & $\mathrm{Ja}$ & k. A. & k. A. \\
\hline Hahnenfuß & Ranunculus spp & $\mathrm{Ja}$ & Nein & $\mathrm{Ja}$ & Ja/k. A. & k. A. \\
\hline Franzosenkraut & Galinsoga parviflora & $\mathrm{Ja}$ & $\mathrm{Ja}$ & Nein & $\mathrm{Ja}$ & Nein \\
\hline Breit- und Spitzwegerich & Plantago spp & $\mathrm{Ja}$ & $\mathrm{Ja}$ & Nein & $\mathrm{Ja} / \mathrm{k} . \mathrm{A}$. & k. A. \\
\hline
\end{tabular}

k. A. keine Angabe 
Abb. 5 Häufigkeit (Anzahl der Boniturrahmen in denen die Beikrautarten nachgewiesen wurden) des Auftretens der Beikräuter bei den 3 Vegetationskartierungen am a 9. Mai vor der Herbizidapplikation, b 11. Juli (zwei Monate nach der Herbizidanwendung) und am c 14 . Oktober 2019 in der Fläche F2 am Campus Klein-Altendorf im Baumstreifen von Apfelbäumen der Sorten 'Golden Delicious' und 'Elstar, Michielsen' a

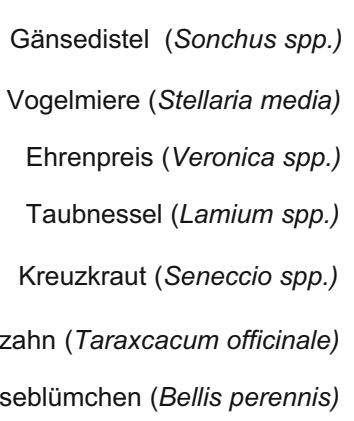

Behaartes Schaumkraut (Cardamine hirsuta)

Gras (Poa spp.)

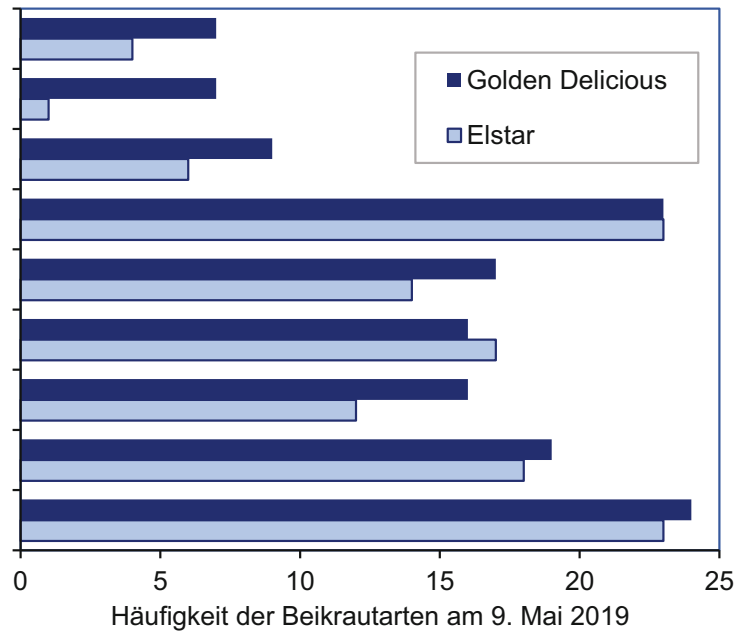

b

Gänsedistel (Sonchus spp.)
Vogelmiere (Stellaria media)
Ehrenpreis (Veronica spp.)
Taubnessel (Lamium spp.)
Kreuzkraut (Seneccio spp.)

Löwenzahn (Taraxcacum officinale)

Gänseblümchen (Bellis perennis)

Behaartes Schaumkraut (Cardamine hirsuta)

Gras (Poa spp.)

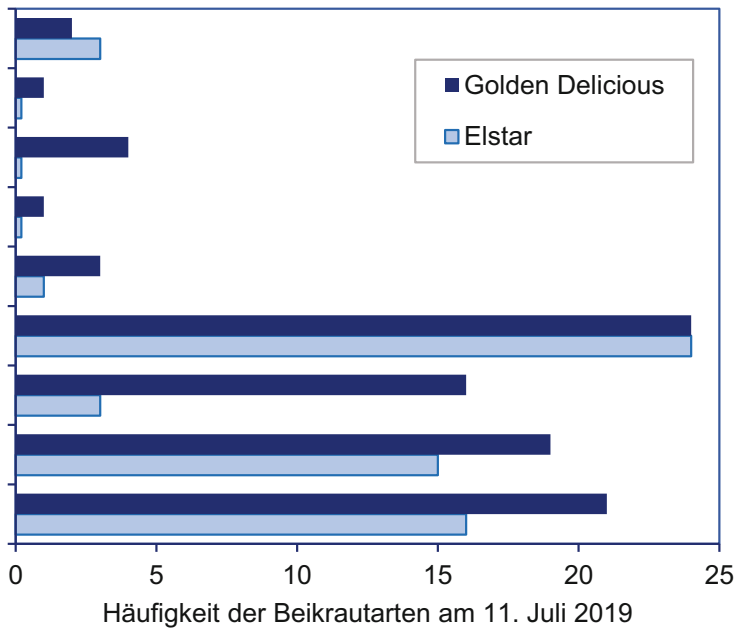

C

Gänsedistel (Sonchus spp.)
Vogelmiere (Stellaria media)
Ehrenpreis (Veronica spp.)
Taubnessel (Lamium spp.)
Kreuzkraut (Seneccio spp.)

Löwenzahn (Taraxcacum offiicinale)

Gänseblümchen (Bellis perennis)

Behaartes Schaumkraut (Cardamine hirsuta)

Gras (Pos spp.)

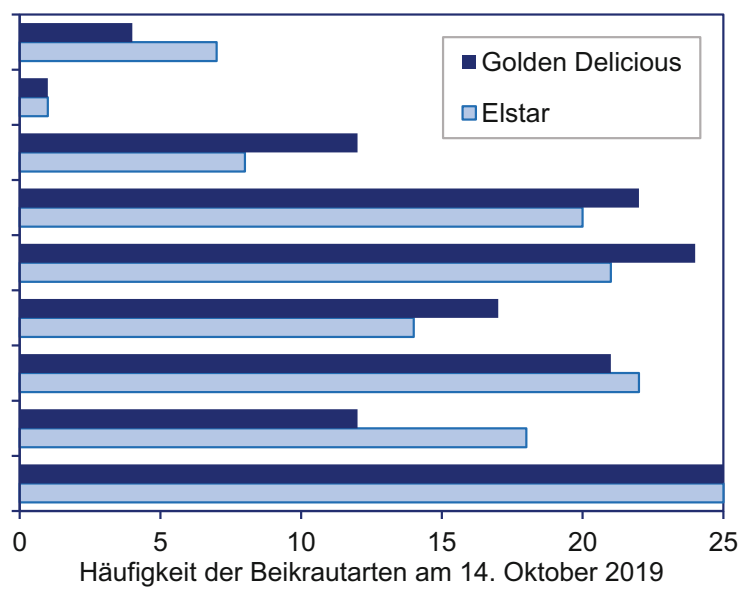

der Sonnenstandswinkel mit $57^{\circ}$ noch geringer war als zur Mittagszeit bei $62^{\circ}$ Sonnenstandswinkel.

Die prozentualen Lichteinstrahlungswerte der Ceptometer-Messungen (10-20\%) im Baumstreifen lagen weit unter den Werten der Schachbrettmethode mit Werten von 40-75\% (Abb. 9); bei beiden Methoden zeigte sich die höhere Beschattung der 'Elstar'-Bäume im Baumstreifen.
Die prozentuale Lichteinstrahlung von ca. $30 \% \mathrm{im}$ Baumstreifen unter den Apfelbäumen war an einem Tag mit diffuser Belichtung im Spätherbst höher als an einem Tag mit direkter Sonneneinstrahlung (ca. 20\%; Abb. 9). Bei dieser Messung konnte kein Unterschied zwischen den beiden Apfelsorten 'Elstar' und 'Golden Delicious' festgestellt werden (Abb. 10) und lässt darauf schließen, 
Abb. 6 a Mittlere Beikrautbedeckung und $\mathbf{b}$ mittlere Wuchsstärke der Beikräuter im Baumstreifen unter Apfelbäumen der Sorten 'Elstar, Michielsen' und 'Golden Delicious' am Campus F2 zum ersten Boniturtermin am 9. Mai 2019 ein Jahr nach der letzten Herbizidbehandlung im Vorjahr. MW \pm SE $(n=18)$ Klein-Altendorf auf der Fläche
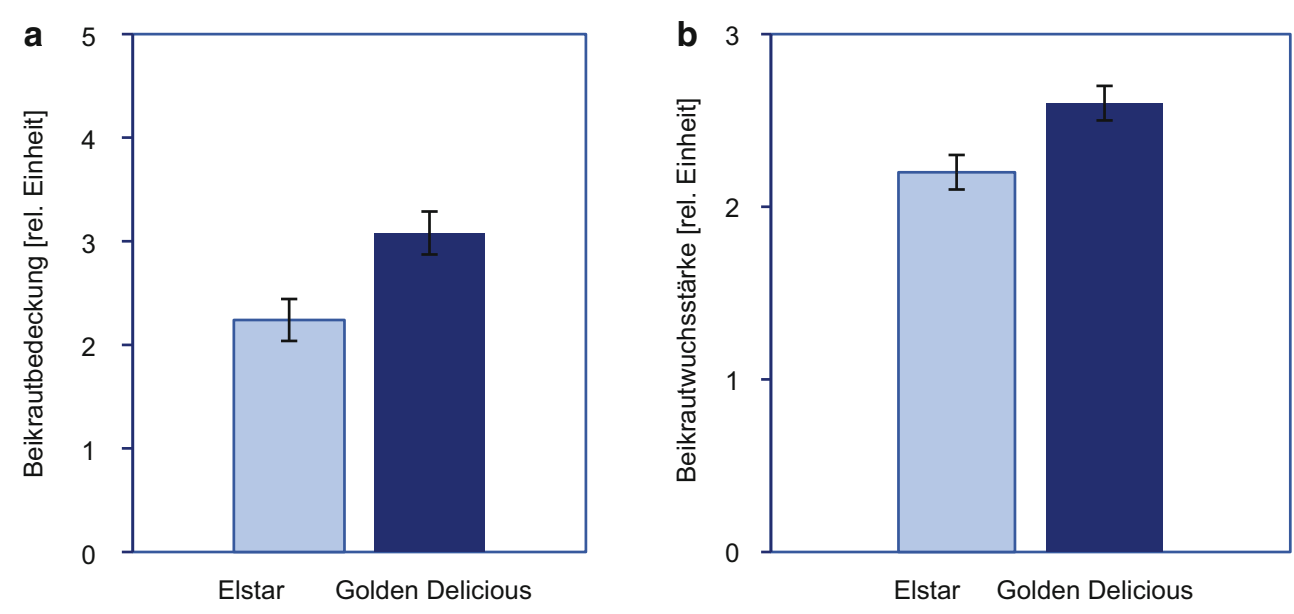

Abb. 7 Mittlere Anzahl der dominierenden Beikrautarten zu drei Boniturterminen im Frühling, Sommer und Herbst in der Vegetationsperiode 2019 in Baumstreifen unter 'Elstar, Michielsen' und 'Golden Delicious' Apfelbäumen in der Parzelle F2. MW \pm SE $(n=25)$

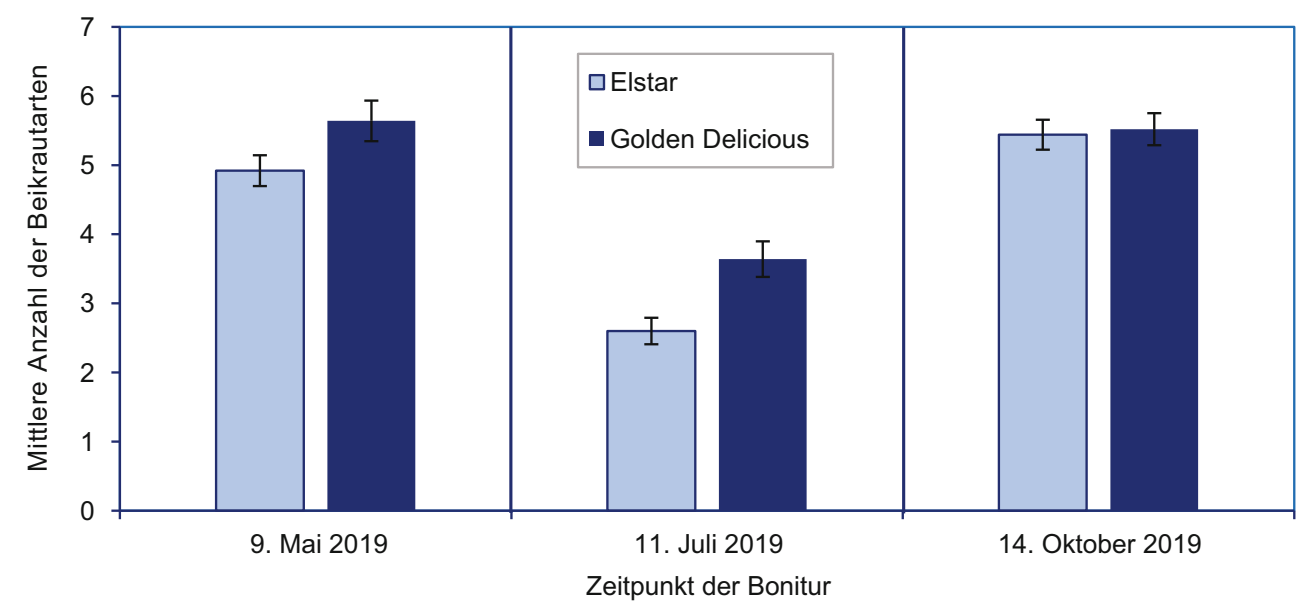

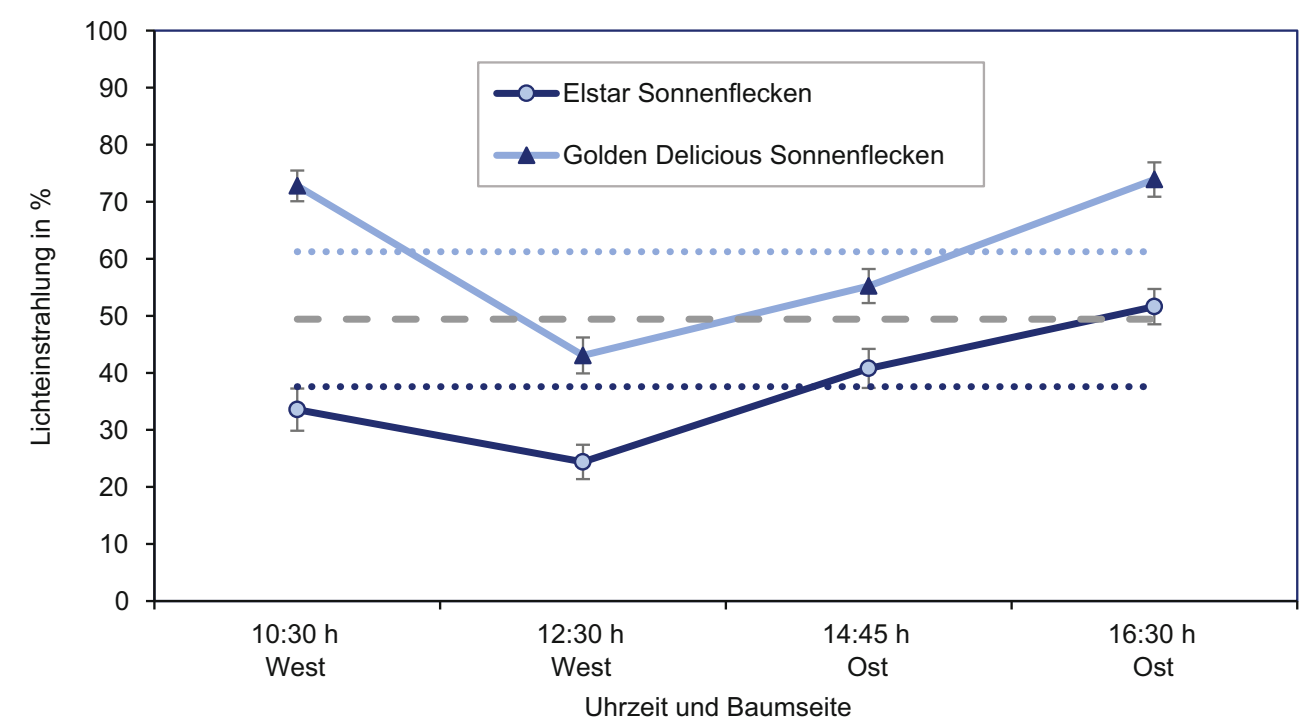

Abb. 8 Tagesverlauf der Lichteinstrahlung auf der Bodenoberfläche ermittelt nach der Schachbrettmethode unter ausgewachsenen 'Elstar, Michielsen'- und 'Golden Delicious'-Apfelbäumen in zwei benachbarten Baumreihen an einem wolkenlosen Tag (24. Juni 2019) am Campus KleinAltendorf (max. Sonnenstandswinkel $62^{\circ}$ ) auf der Fläche J1. Gemessen wurde um 10:30 Uhr und 12:30 Uhr auf der West- und um 14:45 Uhr und um 16:30 Uhr auf der Ostseite der Baumreihe. Die horizontale grau gestrichelte Grade gibt die mittlere Lichteinstrahlung der beiden Sorten an und die gepunkteten blauen Geraden geben den Durchschnitt der einzelnen Sorten an. MW \pm SE $(n=18)$ 
Abb. 9 Lichteinstrahlung in $\%$ auf der Bodenoberfläche unter benachbarten Baumreihen ausgewachsener 'Elstar, Michielsen' und 'Golden Delicious' Apfelbäumen, ermittelt mit der Schachbrett-Methode (durchgezogene Linie) und mit dem Ceptometer (gestrichelte Linie) an einem wolkenlosen Tag mit direkter Sonneneinstrahlung (30. August 2019) am Campus Klein-Altendorf (max. Sonnenstandswinkel $51^{\circ}$ ) auf der Fläche J1. Um 11:00 Uhr und 13:00 Uhr MESZ wurde auf der Westseite der Baumreihe gemessen, um 15:00 Uhr auf der Ostseite. $\mathrm{MW} \pm \mathrm{SE}(n=10)$

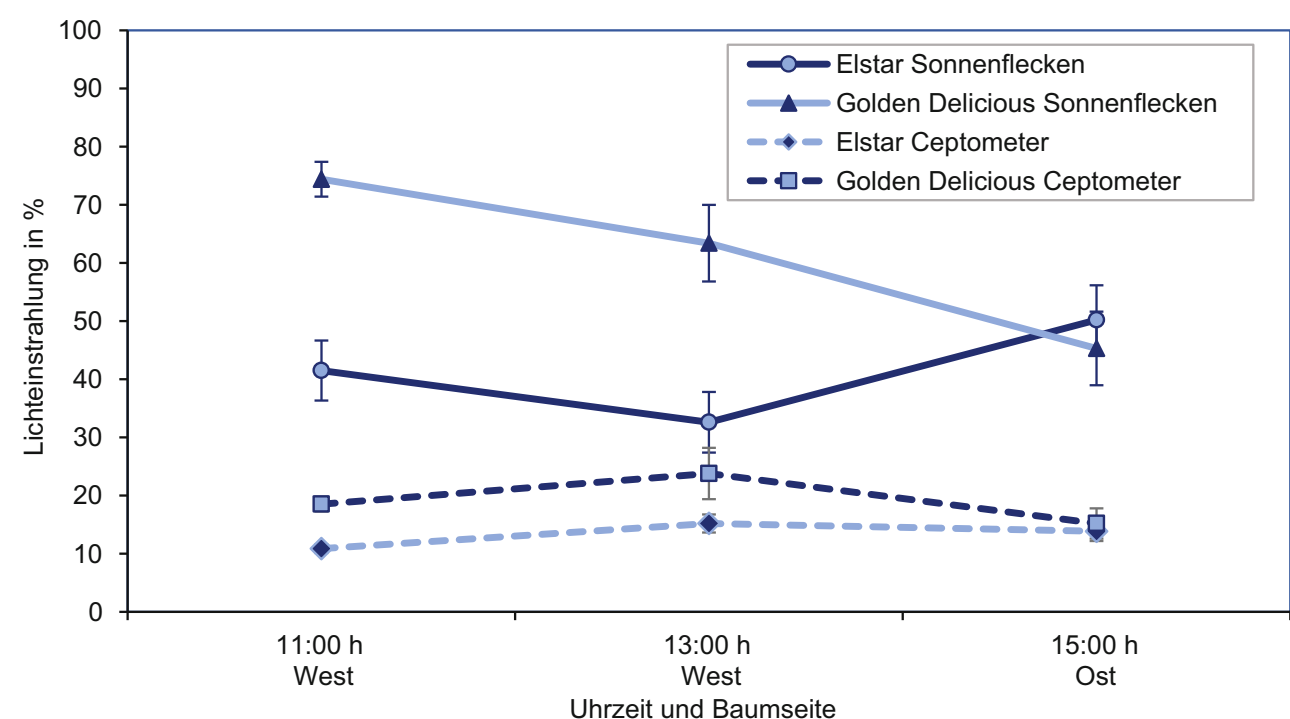

Uhrzeit und Baumseite

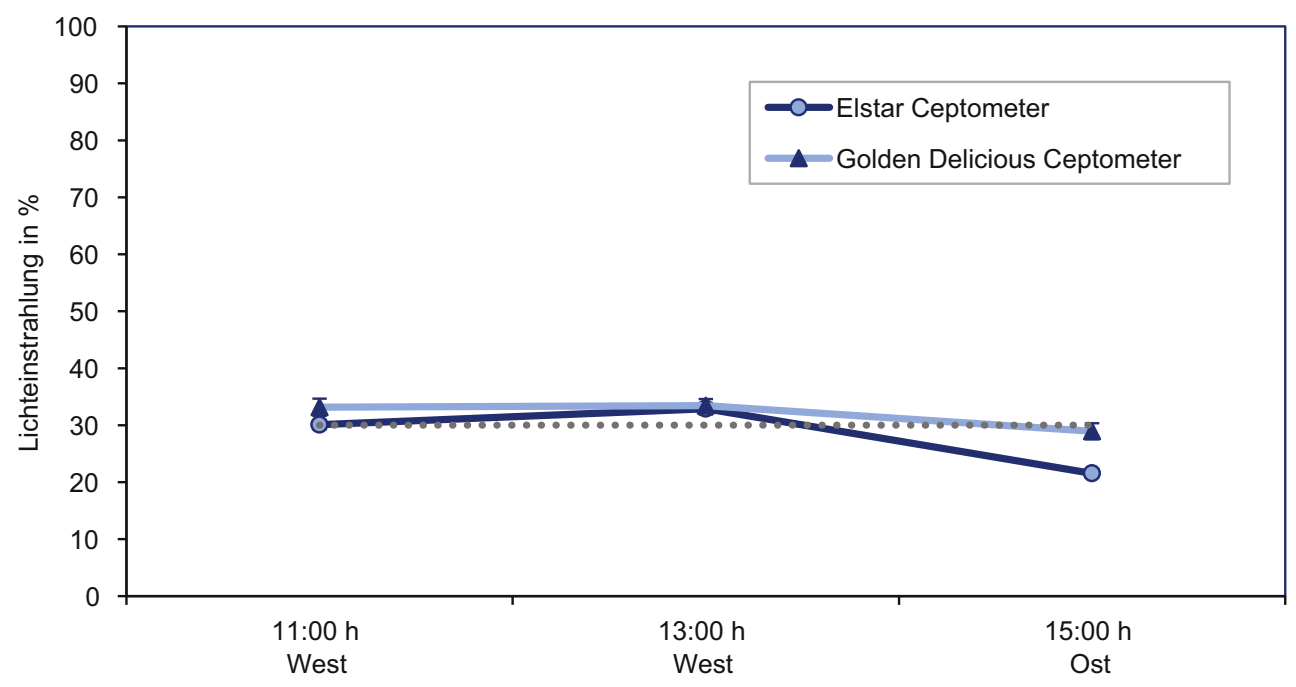

Uhrzeit und Baumseite

Abb. 10 Mit dem Ceptometer ermittelter prozentualer Anteil der Lichteinstrahlung an einem Tag mit diffuser Lichteinstrahlung auf der Bodenoberfläche unter Apfelbäumen der Sorten 'Elstar, Michielsen' und 'Golden Delicious' an der gesamten diffusen Lichteinstrahlung an einem bedeckten Tag (26. September 2019). Die Messungen erfolgten auf der Parzelle J1 am Campus Klein-Altendorf (max. Sonnenstandswinkel $40^{\circ}$ ) unter zwei benachbarten Baumreihen um 11:00 Uhr und 13:00 Uhr auf der Westseite der Baumreihen und um 15:00 Uhr auf der Ost-Seite. Die gepunktete graue Linie gibt den Mittelwert beider Sorten an. MW \pm SE $(n=10)$

dass der beobachtete Effekt der geringeren Verunkrautung durch Beschattung besonders durch Tage mit direkter Sonneneinstrahlung hervorgerufen wird.

\section{Keimtest}

Die Aussaatversuche mit Keimschalen im Baumstreifen beider Apfelsorten ('Golden Delicious' und 'Elstar') im Sommer haben gezeigt, dass die Keimrate des Lichtkeimers Kresse (Lepidium sativum) bei beiden Apfelsorten (ca. $80 \%$ ) identisch zu der des Dunkelkeimers Borretsch (Borago officinalis) (ca. 80\%) war und somit kein Unter- schied in der Keimrate in Abhängigkeit von der Lichtstärke auftrat (Abb. 11).

\section{Diskussion}

Ziel der Arbeit war, mögliche Ursachen und Wirkungsmechanismen dieses Phänomens der natürlichen Unkrautunterdrückung in 'Elstar'-Anlagen im Vergleich zu anderen ausgewachsenen Apfelbäumen auf der gleichen Unterlage am gleichen Standort zu untersuchen. 

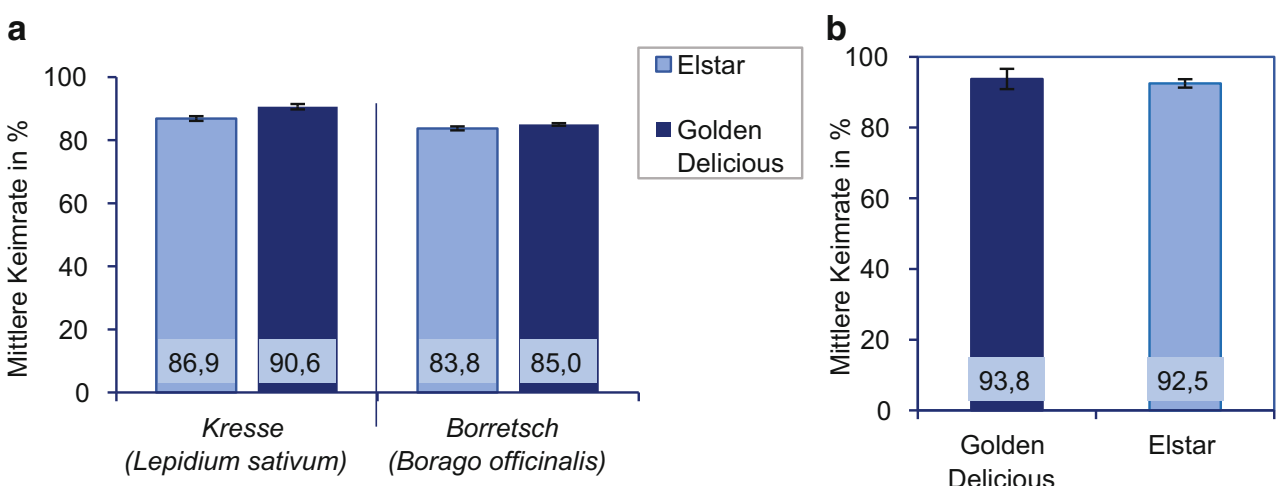

Abb. 11 Keimtests: a Am 30. August 2019 bonitierte Keimrate in \% des Lichtkeimers Kresse (Lepidium sativum) und des Dunkelkeimers Borretsch (Borago officinalis), in Saatgefäßen im Baumstreifen aufgestellt unter Apfelbäumen der Sorten 'Elstar, Michielsen' und 'Golden Delicious'. MW \pm SE ( $n=4$ Saatschalen) und b Keimtest mit Kresse (Lepidium sativum) in Saatschalen mit Substrat und zerkleinertem Falllaub (1:10 v/v) von 'Elstar, Michielsen' und 'Golden Delicious' Apfelbäumen. MW \pm SE ( $n=4$ Saatschalen)

Die Ergebnisse in Tab. 5 bestätigen zum Teil die Beobachtungen der früheren Vegetationskartierung in den Obstanlagen im Rheinland, dass wiederholte Glyphosatanwendung (über 5 bis 10 Jahre) im Meckenheimer Obstbaugebiet einjährige Samenunkräuter, wie Vogelmiere (Stellaria media) und Gemeines Kreuzkraut (Senecio vulgaris) indirekt selektierte, wohingegen Gemeine Quecke (Agropyron repens), Weißes Straußgras (Agrostis stolonifera), Kriechender Hahnenfuß (Ranunculus repens) und Ampfer (Rumex spp.) verdrängt wurden (Nohl 1982). Erstaunlich ist, dass die gleiche selektive Wirkung von Glyphosat (2,5 bzw. $3,6 \mathrm{~kg} / \mathrm{ha} / \mathrm{Jahr}$ ) auf die zwei oben aufgeführten Arten, Vogelmiere (Stellaria media) und Gemeines Kreuzkraut (Senecio vulgaris), und Gräser auch im Weinbau von Stalder et al. (1977) in Rebanlagen in der deutschsprachigen Schweiz beobachtet wurde. Glyphosat unterdrückte in der Schweiz Convolvulus-Arten, verhinderte aber nach der Applikation nicht das Auflaufen von einjährigen Samenunkräutern, wie Vogelmiere (Stellaria media), Gemeines Kreuzkraut (Senecio vulgaris) und Taubnessel (Lamium spp.) (Stalder et al. 1977).

Neben Vogelmiere (Stellaria media) und Kreuzkraut (Seneccio spp.) traten in unseren Bonituren wesentlich stärker Gras (Poa spp.), Gänseblümchen (Bellis perennis), Löwenzahn (Taraxcacum officinale) und Schaumkraut (Cardamie hirsuta) auf. Die höhere Anzahl an durch Glyphosat und MCPA selektierten Arten im vorliegenden Versuch (Abb. 3) könnte durch den selteneren Einsatz der verwendeten Herbizidkombination zum Zeitpunkt der Untersuchung bedingt sein mit einem längeren Zeitabstand von einem Jahr zur Regeneration der betroffenen Beikrautflora. Nach Stalder et al. (1977) beseitigt die erste Glyphosatbehandlung den Vegetationsaufwuchs einmalig, sodass die bereits im Boden vorhandenen Samen der Samenunkräuter (z. B. Vogelmiere) nach der Applikation im Frühjahr als erstes wieder auflaufen können. Gemeine Quecke (Agropyron repens), Weißes
Straußgras (Agrostis stolonifera), Kriechender Hahnenfuß (Ranunculus repens) und Ampfer (Rumex spp.) wurden zum Zeitpunkt der Bonitur auf den untersuchten Parzellen am Campus Klein-Altendorf nicht aufgefunden. Bei den vorangegangenen Vegetationskartierungen im Meckenheimer Raum waren die untersuchten Apfelsorten bei der Auswertung nicht berücksichtigt. Die Kombination der Ergebnisse von Kolbe (1969), Nohl (1982) und Stalder et al. (1977) lässt den Schluss zu, dass dieses Phänomen der Unkrautunterdrückung im Baumstreifen von Elstar-Anlagen sortenspezifisch ist und eine Kombination aus den Folgen der stärkeren Beschattung dieser Sorte bzw. Lichtmangel und Herbizid- bzw. Glyphosatanwendung (und spezifischer Wirkstoffe). Die Unterlage spielt vermutlich keine Rolle, da die Vergleichspflanzen immer auf derselben Unterlage (M 9 in Klein-Altendorf bzw. M2 am Laacherhof bei Burscheid) standen.

Anhand der Ergebnisse des zweiten Keimtests konnte keine direkte keimhemmende Wirkung der Apfelbäume oder des Falllaubes der Sorte 'Elstar' nachgewiesen werden (Abb. 11) - im Gegensatz zu z.B. Walnuss (Juglans regia), aus deren Blättern herbizidwirksame Stoffe extrahiert wurden und als Herbizide im ökologischen Anbau gegen ausgesuchte Beikräuter verwendet werden könnten (Kocaçaliskan und Terzi 2001).

Die Keimversuche haben gezeigt, dass die Keimrate des Lichtkeimers Kresse (Lepidium sativum) (ca. 80\%) identisch zu der des Dunkelkeimers Borretsch (Borago officinalis) (ca. 80\%) war und somit kein Unterschied in der Keimrate in Abhängigkeit von der Lichtstärke auftrat (Abb. 11). Bei ansonsten günstigen Keimbedingungen (Wasserversorgung, Temperatur) kann es vorkommen, dass Licht- und Dunkelkeimer unabhängig von der herrschenden Lichteinstrahlung gleich gut keimen (Kuhn 1916). 


\section{Fazit}

Die Kombination unserer Ergebnisse (Abb. 7, 8 und 9 mit 'Elstar Michielsen' auf M 9) mit denen von Kolbe (1969) (mit 'Ingrid Marie' auf M 2) lassen vermuten, dass die beikrautunterdrückende Wirkung im Wiederaufwuchs nach Beikrautbekämpfung bei ausgewachsenen 'Elstar, Michielsen'-Apfelbäumen sortenbedingt durch die Schattierung dieser wüchsigeren Bäume und möglicherweise anderer Faktoren bedingt ist und nicht von der Bewirtschaftungsform (IP oder Bio) abhängt.

Danksagung Wir danken Dr. Onno Muller von der Forschungsanlage Jülich für das Überlassen des Ceptometers, Dr. Adrian Engel, Pflanzenschutzdienst der Landwirtschaftskammer für Durchsicht des Manuskriptes und Dr. Dereck P. Hucklesby, Bristol, UK für die Korrektur des englischen Abstract.

Funding Open Access funding enabled and organized by Projekt DEAL.

Interessenkonflikt A. Engel, A. Kunz und M. Blanke geben an, dass kein Interessenkonflikt besteht.

Open Access Dieser Artikel wird unter der Creative Commons Namensnennung 4.0 International Lizenz veröffentlicht, welche die Nutzung, Vervielfältigung, Bearbeitung, Verbreitung und Wiedergabe in jeglichem Medium und Format erlaubt, sofern Sie den/die ursprünglichen Autor(en) und die Quelle ordnungsgemäß nennen, einen Link zur Creative Commons Lizenz beifügen und angeben, ob Änderungen vorgenommen wurden.

Die in diesem Artikel enthaltenen Bilder und sonstiges Drittmaterial unterliegen ebenfalls der genannten Creative Commons Lizenz, sofern sich aus der Abbildungslegende nichts anderes ergibt. Sofern das betreffende Material nicht unter der genannten Creative Commons Lizenz steht und die betreffende Handlung nicht nach gesetzlichen Vorschriften erlaubt ist, ist für die oben aufgeführten Weiterverwendungen des Materials die Einwilligung des jeweiligen Rechteinhabers einzuholen.

Weitere Details zur Lizenz entnehmen Sie bitte der Lizenzinformation auf http://creativecommons.org/licenses/by/4.0/deed.de.

\section{Literatur}

Braun-Blanquet J (1964) Pflanzensoziologie: Grundzüge der Vegetationskunde, 3. Aufl. Springer, Berlin

Engel G (1968) Herbizidspritzungen als Bodenpflegemaßnahmen im Obstbau. Erwerbs-Obstbau 10:6-9

Kocaçaliskan I, Terzi I (2001) Allelopathic effects of walnut leaf extracts and juglone on seed germination and seedling growth. J Hortic Sci Biotechnol 76(4):436-440

Kolbe W (1969) Die Anwendung von Herbiziden als Maßnahme der Bodenpflege und des Pflanzenschutzes in ihrem Einfluss auf Wuchsleistung, Ertrag und Fruchtqualität im Kernobstbau. Erwerbs-Obstbau 5:81-86

Kuhn E (1916) Dunkelkeimer und Substrat. Ber Dtsch Bot Ges 34:369-386

Lepsis J, Blanke M (2001) Lichtausnutzung und Stammquerschnitt als Maßstäbe für die prognostische Bewertung von Pflanzsystemen bei Apfel. Erwerbs-Obstbau 43:142-150

Lepsis J, Blanke M (2005) Ökotoxikologische Wirkungen von Teeröl-imprägnierten Holzpfählen. Z Umweltwiss Schadstoffforsch 17:13-19. https://doi.org/10.1007/BF03038689.pdf (UWSFESPR)

Nohl C (1982) Auswirkungen eines langjährigen einseitigen Herbizideinsatzes auf die Unkrautflora in Obstanlagen. Dissertation, Rheinische Friedrich-Wilhelms-Universität Bonn

Palmer JW, Jackson JE (1977) Seasonal light interception and canopy development in hedgerow and bed system apple orchards. J Appl Ecol 14:539

Stalder L, Potter CA, Barben E (1977) Neue Erfahrungen mit integrierten Maßnahmen zur Bekämpfung der Acker- und Zaunwinde (Convolvulus arvensis, C. sepium) im Weinbau. In: Proc. EWRS Symposium Uppsala: Methods of weed control and their integration, Bd. 1, S 221-228

Statistisches Bundesamt (2017) Land- und Forstwirtschaft, Fischerei: Landwirtschaftliche Bodennutzung - Baumobstflächen 2017. Destatis, Wiesbaden (Fachserie 3 Reihe 3.1.4)

Wagenmakers PS, Tazelaar M (1999) Modelling light interception on the basis of sunfleck measurements. Acta Hort 499:297-303

Warren Wilson J (1960) Inclined point quadrats. New Phytol 59:1-8

Werth J, Beck M, Wilhelm U, Kittemann D (2019) Einfluss verschiedener Maßnahmen zur Baumstreifenpflege im Apfelanbau auf Bodenklima und Unkrautwachstum. BHGL-Tagungsband, Bd. 34, S 77

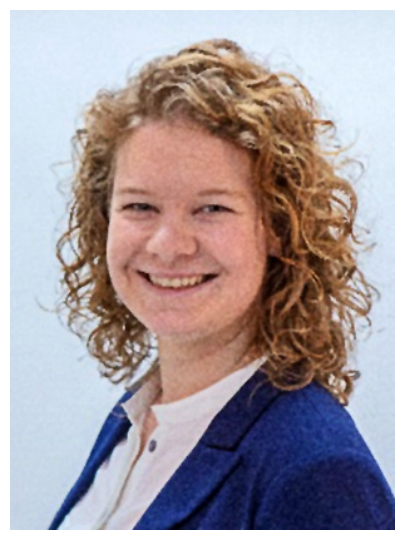

Antonia Engel studierte seit 2013 Agrarwissenschaften mit dem Schwerpunkt Nutzpflanzenwissenschaften an der Universität Bonn und schloss ihr Studium im Dezember 2019 mit dem Master-Nutzpflanzenwissenschaften ab. Ihr besonderes Interesse gilt dem Obstbau und der Qualitätssicherung. 